\title{
Hadron Spectrum from Lattice QCD
}

\author{
S. Dürr ${ }^{1,2, a}$ \\ 1 Bergische Universität Wuppertal, Gaussstr. 20, D-42119 Wuppertal, Germany \\ 2 Jülich Supercomputing Center, Forschungszentrum Jülich, D-52425 Jülich, Germany
}

\begin{abstract}
In order to claim that we understand strong interactions quantitatively, we should be able to derive key properties of hadrons, such as their mass, "ab initio" from the interaction of quarks and gluons. In particular the phenomenon of "dimensional transmutation", i.e. of having a massive proton even with exactly massless quarks and gluons, is a distinctive property of QCD. In this talk I summarize recent efforts, in the context of the Budapest-Marseille-Wuppertal collaboration, to compute the masses of the flavor non-singlet mesons and of the light baryon octet and decuplet in the isospin limit of QCD on the lattice, with fully controlled systematics.
\end{abstract}

\section{Introduction}

The bulk part of the visible matter in the universe is made from protons and neutrons. The modern theory of strong interactions, known as Quantum Chromodynamics (QCD), describes how quarks and gluons amalgamate to form nucleons and other strongly interacting states, called hadrons. Due to the highly non-linear structure of these interactions, any form of ab-initio determination of the properties of these hadrons from the underlying interaction of quarks and gluons has remained a challenge for decades. The goal of this contribution is to describe how lattice QCD tackles the problem and allows us to obtain results with fully controlled systematics.

At first sight QCD is rather analogous to QED, the quantum theory of relativistic electrodynamics. This is because the Lagrangian (in Euclidean space) [1]

$$
L_{\mathrm{QCD}}=\frac{1}{2} \operatorname{Tr}\left(F_{\mu \nu} F_{\mu \nu}\right)+\sum_{i=1}^{N_{f}} \bar{\psi}\left(D+m_{i}\right) \psi
$$

closely resembles its QED counterpart. The difference is that the field-strength tensor $F_{\mu \nu}=\partial_{\mu} A_{v}-\partial_{\nu} A_{\mu}+\mathrm{i} g\left[A_{\mu}, A_{\nu}\right]$ is (for a given $\mu, v$ combination) a $3 \times 3$ matrix in "color" space with a commutator term (which in the case of QED is zero). The symbol $\operatorname{Tr}($.) refers to the trace over this internal degree of freedom. A similar sum is implied in the second term of (1) which, in addition, contains an explicit sum over the "flavors" one wants to deal with, usually "up" $(u)$, "down" $(d)$ and "strange" $(s)$. This commutator term and the pertinent non-linearities render QCD a fascinating theory, but also a demanding one. One key property is called "asymptotic freedom" [2,3]. It states that the effective (renormalized) version of the coupling $g$ showing up in $F_{\mu \nu}$ becomes weak at short distances. In other words, at high energies (or high relative momentum) quarks and gluons interact weakly, allowing for standard perturbative

\footnotetext{
a e-mail: s.durr (AT) fz-juelich.de
}

methods to be used. By contrast, at low relative momentum the interaction becomes strong, to the point where asymptotic states do not carry a net "color" charge any more. This phenomenon is called "confinement".

In order to derive these properties from the QCD Lagrangian (1), the theory needs to be regularized, both in the ultraviolet and in the infrared. A particularly convenient choice is the lattice, as introduced by Wilson $[4,5]$. The finite lattice spacing $a$ and the finite four-dimensional box volume $V=L^{4}$ provide such a regularization. In other words, they make sure that the generating functional

$$
\begin{aligned}
& Z[\bar{\eta}, \eta]= \\
& Z_{0} \int D U D \bar{\psi} D \psi e^{-\int S_{g} d x-\sum \int[\bar{\psi}(D+m) \psi+\bar{\eta} \psi+\bar{\psi} \eta] d x}= \\
& Z_{0} \int D U \prod \operatorname{det}(D+m) e^{+\sum \int \bar{\eta}(D+m)^{-1} \eta d x} e^{-\int S_{g} d x}
\end{aligned}
$$

(with sums and products running over the flavors) is welldefined, and the important property of gauge symmetry is maintained exactly. The task is then to compute a given dimensionless ratio, e.g. $M_{N} / M_{\Xi}$, for a series of lattice spacings $a$, box sizes $L$ and quark masses $m_{u}, m_{d}, m_{s}$, and to perform a well monitored extrapolation of this ratio to zero lattice spacing, infinite volume and physical values of the quark masses. If one can control all sources of error in each step, one can claim to have an ab-initio result for the strong interactions which provide the bulk part of the mass of the visible matter in the universe.

\section{Elements of Lattice Gauge Theory}

Putting a non-gauge field theory on the lattice is straightforward. One lets the field $\phi$ reside on a $d$-dimensional lattice $\left(x_{1}, \ldots, x_{d}\right)$ with $x_{\mu} \in\left\{a, 2 a, \ldots, N_{\mu} a\right\}$ and periodic or anti-periodic boundary conditions in all directions. Furthermore, one replaces derivatives $\left(\partial_{\mu} \phi\right)(x)$ by discrete differences $(\phi(x+a \hat{\mu})-\phi(x-a \hat{\mu})) /(2 a)$, where $\hat{\mu}$ denotes the 
unit-vector in $\mu$-direction. This recipe provides a lattice formulation of a $\phi^{4}$-type field theory, which allows one to address the triviality issue pertinent to such a theory.

With gauge theories the situation is a bit more involved, as a naive application of this recipe would break gaugeinvariance through $O(a)$-suppressed terms [4]. It was Wilson who noted that one can maintain gauge-invariance exactly, if one lets only the matter fields $\phi$ (bosonic) and $\psi$ (fermionic) reside on the sites $x$, while the gauge field $A_{\mu}(x)$ lives on the links connecting the neighboring sites $x$ and $x+\hat{\mu}$. More precisely, it is the path-ordered exponential $U_{\mu}(x)=\exp \left(\mathrm{i} g \int A_{\mu}(\tau) d \tau\right)$ which is considered the fundamental object [4]. Here, the integral runs from $x$ to $x+a \hat{\mu}$, and $U_{\mu}(x)$ is a $U(1)$ group element in QED or a $S U(3)$ group element in QCD, respectively.

Adding fermions makes things again a bit more tricky. That Grassmannian numbers cannot be directly represented through ordinary (complex) variables is not a problem, it simply means that the functional determinant appears in (2), and not it's inverse. The more severe part is that the straightforward (so-called "naive") fermion discretization yields $2^{d}$ species, not just one. In [5] Wilson provides a clear solution to this so-called "doubling problem" which, however, comes at the price of destroying chiral symmetry through $O(a)$ lattice artefacts. Later it was realized that this is a specific manifestation of a general theorem which excludes that a simple (read: ultra-local) action would simultaneously be undoubled and chiral $[6,7]$.

Perhaps the most important feature of Wilson's formulation of QCD $[4,5]$ is that confinement follows as a disorder phenomenon in the strong-coupling limit (see e.g. [8] for a review), and the task is thus to show that this feature persists in the opposite (weak coupling) limit $a \rightarrow 0$. Last but not least, from an analytical point of view this formulation is a bit uneasy, since it breaks Lorentz symmetry, thus giving rise to a plethora of terms.

\section{Choice of Lattice Action and Algorithm}

Creutz was the first author to notice [9] that well established importance sampling techniques from statistical mechanics can be used to calculate specific ratios of QCD functional integrals in Euclidean space on the computer. With this technique and the gluon and fermion discretizations given in $[4,5]$ one can in principle carry out Wilson's program. In practice things look somewhat different. The reason is that the cost, in terms of CPU time, grow rather dramatically along each one of the directions $a \rightarrow 0$, $V \rightarrow \infty, m_{u, d} \rightarrow m_{u, d}^{\text {phys }}$ we aim for.

To mitigate this cost growth some theory investment is required. Let me discuss how the continuum limit $a \rightarrow 0$ is accelerated, postponing the other two steps for a moment. The conceptual background for controlling the costs of the continuum limit has been laid out by Symanzik $[10,11]$. The starting point is that on the lattice any dimensionless ratio differs from its value in the continuum by terms which scale with a certain power of $a$. For the nucleon-to-xi mass ratio considered before, this means that

$$
\frac{M_{N}}{M_{\Xi}}(a)=\frac{M_{N}}{M_{\Xi}}(0)+\operatorname{const}\left(\frac{a}{\Lambda}\right)^{n}+O\left(\left(\frac{a}{\Lambda}\right)^{n+1}\right)
$$

where $n$ is a property of the action used to sample the phase space and to construct the interpolating fields of the states considered, and $\Lambda$ is a QCD intrinsic scale (e.g. $\Lambda_{\mathrm{QCD}}$ or the nucleon mass in the chiral limit).

The crucial observation by Symanzik was that one may augment a given lattice action by "irrelevant" terms (i.e. terms of mass-dimension 5 and higher) with coefficients tuned such that "const" in the first sub-leading term of (3) is zero. This has an enormous impact on the cost, in terms of CPU time, of a physical result in the continuum, because it enlarges the smallest (and hence most expensive) lattice spacing at which simulations need to be carried out. For illustration, let us assume the scaling regime [the region where the $O\left((a / \Lambda)^{n+1}\right)$ term in (3) can be neglected] starts at $0.1 \mathrm{fm}$, and we wish to have data over $3 / 4$ of the range in which we extrapolate linearly. In a theory with $O(a)$ cut-off effects, we would sample at $a / \mathrm{fm} \simeq 0.025,0.05,0.075,0.1$, while in a theory with $O\left(a^{2}\right)$ effects we would use $a / \mathrm{fm} \simeq$ $0.05,0.071,0.087,0.1$. This factor of 2 on the smallest lattice spacing amounts to great savings.

Following these general ideas the Budapest-MarseilleWuppertal collaboration uses the Symanzik action [12]

$$
\begin{array}{r}
S_{g}=\frac{\beta}{3} \sum_{x}\left\{c_{0} \sum_{1 \leq \mu<v \leq 4}\left[1-\operatorname{Re} \operatorname{Tr}\left(U_{x, \mu v}^{1 \times 1}\right)\right]+\right. \\
\left.c_{1} \sum_{1 \leq \mu \neq v \leq 4}\left[1-\operatorname{Re} \operatorname{Tr}\left(U_{x, \mu v}^{1 \times 2}\right)\right]\right\}
\end{array}
$$

for the gluons and the Sheikholeslami-Wohlert action [13]

$$
\begin{aligned}
S_{f}=a^{4} \sum_{x}\{ & \frac{1}{2 a} \sum_{\mu=1}^{4} \bar{\psi}_{x}\left[\left(\gamma_{\mu}-I\right) V_{x, \mu} \psi_{x+\hat{\mu}}\right. \\
& \left.\left.-\left(\gamma_{\mu}+I\right) V_{x-\hat{\mu}, \mu}^{\dagger} \psi_{x-\hat{\mu}}+\left(\frac{4}{a}+m\right) \psi_{x}\right]\right\} \\
- & a^{5} \sum_{x}\left\{\frac{c_{\mathrm{SW}}}{2} \sum_{\mu<v} \bar{\psi}_{x} \sigma_{\mu \nu} F_{x, \mu v}[V] \psi_{x}\right\}
\end{aligned}
$$

for the quarks, with $\sigma_{\mu \nu}=(\mathrm{i} / 2)\left[\gamma_{\mu}, \gamma_{\nu}\right]$ and hermitean $F_{\mu \nu}$. The improvement coefficients are set to their tree-level values, i.e. $c_{1}=-1 / 12, c_{0}=1-8 c_{1}=5 / 3$ and $c_{\mathrm{SW}}=1$. Note that in (4) the original ("thin") gauge links $U_{x, \mu}$ are used to define the $1 \times 1$ and $1 \times 2$ Wilson loops $U_{x, \mu \nu}^{1 \times 1}$ and $U_{x, \mu \nu}^{1 \times 2}$, whereas in (5) modified ("fat") gauge links $V_{x, \mu}$ are used which relate to the $U_{x, \mu}$ through 6 levels of stout smearing [14]. This is a legal modification with an enormous impact on both the stability of the simulation and the speed (see below). Note that the usual ultra-locality of Wilson fermions $D(x, y)=0$ for $\|x-y\|>a$ is maintained, but the ultralocality with respect to changes in the gauge field is replaced by a locality in the modern sense. By this we mean that $\delta(D(x, y)) / \delta(A(z))$ falls off exponentially as $z$ moves away from $x=y$, see [15] for details.

The cost of a QCD simulation with the HMC algorithm [16] is dominated by frequently solving sparse systems of the form $(D+m)^{\dagger}(D+m) x=b$, to keep track of the 

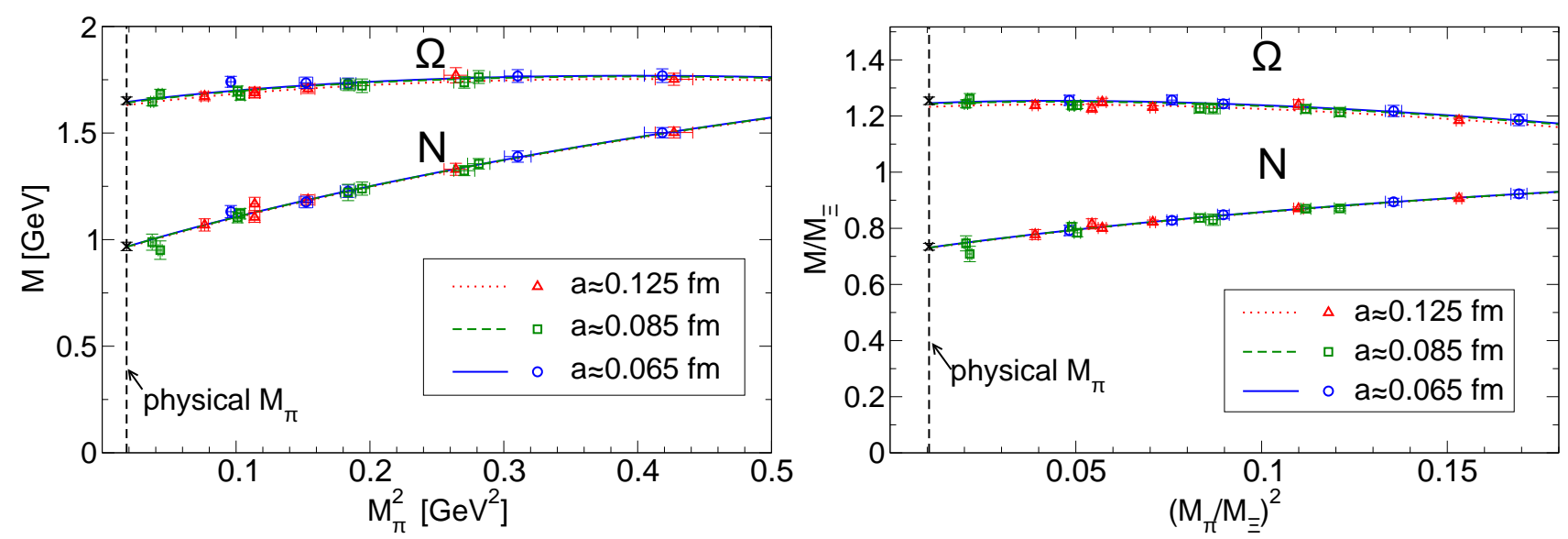

Fig. 1. Synopsis of the quark mass extrapolation of our data to the physical value of $m_{u d}$ (equivalently to the physical value of $M_{\pi}$ ). In the left plot a mass-independent scale setting is used (through $a M_{\Xi}$ at the physical mass point), in the right plot only dimensionless ratios are considered (i.e. the scale is set through $a M_{\Xi}$ in the actual simulation). As expected, the resulting predictions at the physical mass point are fully consistent. Our data at three lattice spacings seem to lie on a universal line, confirming the good scaling seen in [22].

change of the functional determinant in (2). There is a battery of tricks to ease this task. We use even-odd preconditioning [17], multiple time-scale integration [18] ("SextonWeingarten scheme"), multiple mass preconditioning [19] ("Hasenbusch trick"), Omelyan integrator [20], RHMC for the strange quark [21] and mixed-precision solver [22]. As noted in the literature $[23,24]$ combining several of these ingredients yields a dramatic reduction of the critical slowing down that has traditionally been observed for light quark masses.

\section{Algorithm Stability and Scaling Tests}

Having specified our action and algorithm, it is important to verify that it entails a nice scaling of physical observables towards the continuum and - a point particularly relevant for (improved) Wilson fermions - algorithmic stability. We have performed several stability analysis [22], including a hysteresis test (to detect a nearby bulk phase transition) and a determination of the smallest (in magnitude) eigenvalue of the hermitean Wilson operator $H_{m}=$ $\gamma_{5}(D+m)$. The latter test shows how the distribution of $\lambda_{\text {min }}$ gets narrower, at a fixed physical quark mass, as the lattice spacing diminishes. In practice, it is far more convenient to monitor the inverse iteration count $1 / n_{\mathrm{CG}}$ of the solver [22], since this quantity is closely related to $\lambda_{\min }$ and comes for free. An explicit example from a $N_{f}=2+1$ run (with a degenerate quark mass $m_{u d}$ for the first two flavors and a heavier $m_{s}$ for the third one) at $M_{\pi}=190 \mathrm{MeV}$ has been shown in [22]. It indicates a clear gap, providing strong evidence for the stability of the algorithm. We monitor $1 / n_{\mathrm{CG}}$ in all our production runs.

Furthermore, in [22] various scaling tests have been carried out, mostly for $N_{f}=3 \mathrm{QCD}$ at a common quark mass which roughly corresponds to the physical strange quark mass. Both the nucleon $(N)$ and the delta $(\Delta)$ mass have been found to scale rather well, that is the term proportional to $a / \Lambda$ in (3) seems to be absent and the slope [i.e. the prefactor of the $(a / \Lambda)^{2}$ term] seems to be small. We find it remarkable that these properties hold true out to lattice spacings around $a \sim 0.16 \mathrm{fm}$.

\section{Ab-initio calculation of Hadron Masses}

As mentioned in the introduction, one of the classic goals of lattice QCD is to get the spectrum of the light hadrons correct. To provide an ab-initio calculation, such a study should simultaneously satisfy the following requirements:

1. Inclusion of the $u, d$, and $s$ quarks in the fermion determinant with an exact algorithm.

2. A complete determination of the masses of the light flavor-nonsinglet pseudoscalar and vector mesons, as well as the baryon octet and decuplet.

3. Large box volumes to guarantee small finite-size effects on one-particle states and, in addition, at least one data point at an even larger volume to confirm the smallness of these effects.

4. Controlled interpolations and extrapolations of all results to the physical values of the quark masses $m_{s}$ and $m_{u d}=\left(m_{u}+m_{d}\right) / 2$, respectively.

5. Controlled extrapolations to the continuum $(a \rightarrow 0)$, requiring that the data are generated at no less than three values of the lattice spacing $a$.

I would like to stress that important progress on each individual point has been made by a number of groups, but a simultaneous fulfillment of all these requirement has been elusive prior to [15]. As the first and the last point have already been discussed in the previous section, I concentrate on the remaining three.

Regarding point 2, I just mention that particles with a significant admixture of flavor-singlet states have been omitted, since they require a dedicated all-to-all propagator technique and massive I/O capabilities which (at the time when these simulations were performed) were not available on $\mathrm{BG} / \mathrm{L}$ and $\mathrm{BG} / \mathrm{P}$. 


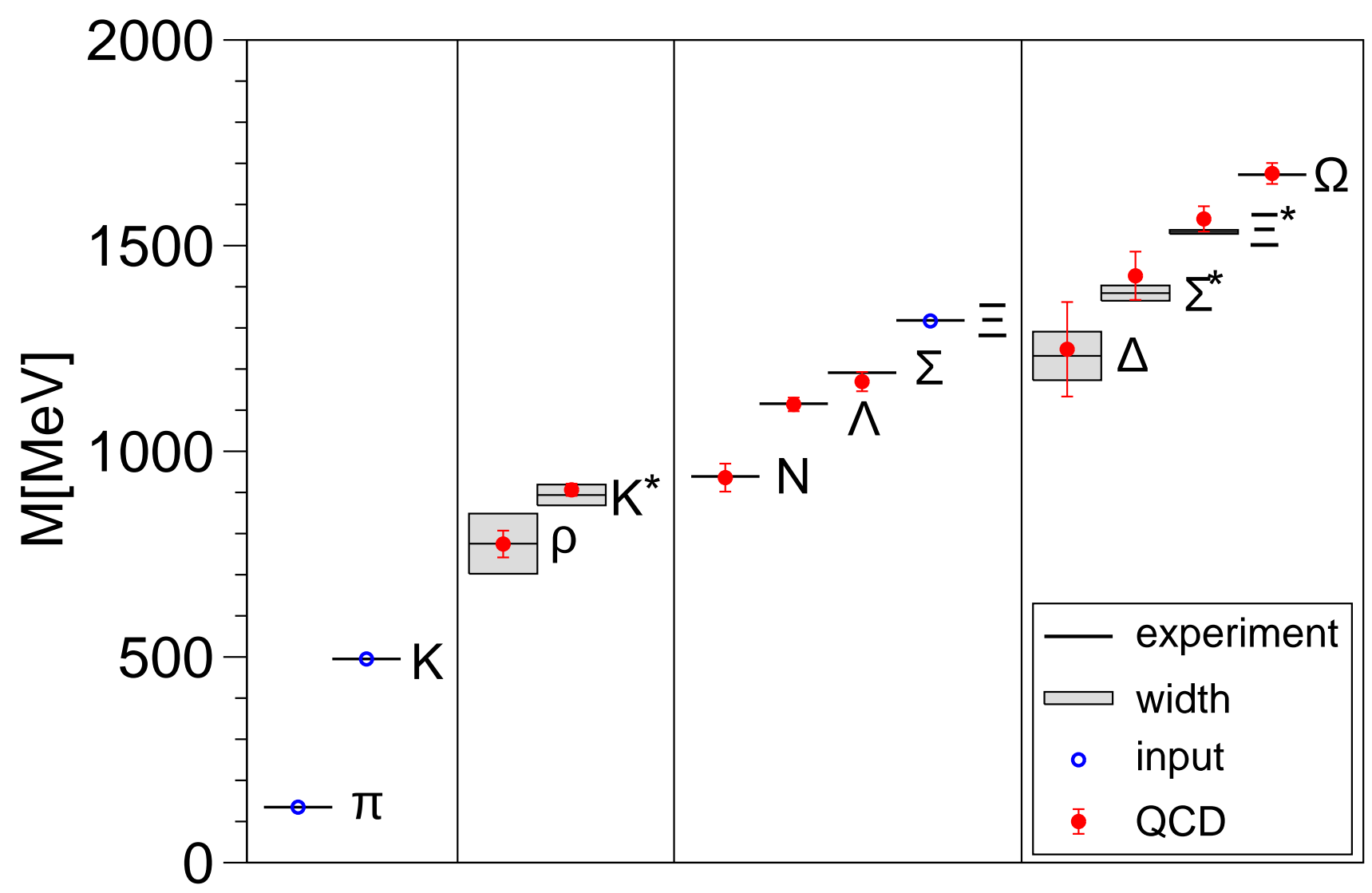

Fig. 2. Our ab-initio calculation [15] of the light hadron spectrum with three light flavors (pseudoscalar and vector meson octet, baryon octet and decuplet, excluding states with significant flavor singlet admixture), compared to experiment. In the latter case states which decay under strong interactions are shown with their width. The error-bars on our data include statistical and systematic uncertainties. Note that $M_{\pi}, M_{K}, M_{\Xi}$ have no error-bar, since they have been used as input to adjust the quark masses and to set the lattice spacing.

Regarding point 3, let me emphasize that the effect of the finite spatial volume has been corrected for, even though these effects were tiny. In particular for two stable states (the pion and the nucleon) the $M(L) / M$ predictions of Chiral Perturbation Theory [25,26] have been tested by augmenting our dataset by dedicated simulations with a smaller and larger $L$. We found complete agreement between our data and the prediction.

Regarding point 4, two comments are in order. The first one concerns the fact that the difference $m_{d}^{\text {phys }}-m_{u}^{\text {phys }}$ is small compared to the typical scale in hadron physics. The effects due to this splitting are tiny compared to our errorbars, and a similar statement holds true for the effects due to the electromagnetic interactions of the quarks (which we do not attempt to simulate on the lattice). This implies that we need to correct the physical values of $M_{\pi}, M_{K}, M_{\Xi}$ to which we want to tune our simulation, for the influence of isospin breaking and electromagnetic effects. On this point we follow [27] and chose $M_{\pi}=135 \mathrm{MeV}, M_{K}=495 \mathrm{MeV}$ and $M_{\Xi}=1318 \mathrm{MeV}$ as input values. The fact that each one of these values comes with an error of a few $\mathrm{MeV}$ is accounted for and contributes to the final systematic error. The second comment concerns the details of the quark mass extrapolation and the scale setting. As one can see in Fig. 1, our data, when plotted against $M_{\pi}^{2}$, show a rather small amount of curvature. Moreover, that our pion mass reaches down to $190 \mathrm{MeV}$ implies that we need to extrapolate very little to reach the target value of $135 \mathrm{MeV}$. We do this both via a Taylor fit and via formulae from Chiral Perturbation Theory [28]. In a similar sense the scale is set either through the value of $a M_{\Xi}$ at the (extrapolated) physical mass point or through the value of $a M_{\Xi}$ in the actual simulation. Both methods are shown in Fig. 1, and the results at the physical mass point are in complete agreement. Similarly, one may choose to set the scale through the $\Omega$ rather than the $\Xi$, which, again, yields consistent results at the physical mass point.

The last two options are, in fact, just two examples of the overall analysis strategy adopted in [15]. Whenever there are several reasonable ways of how to set a given variable to its physical value, we do not just use one of them, but we use them all in a systematic form. Each time we perform a full analysis, including an assessment of the statistical error via a complete bootstrap procedure, leading to an overall distribution of the central values. From this distribution we draw, in the final phase of the analysis, our central value and the best estimate of the overall theoretical or systematic uncertainty. This uncertainty is then added (in quadrature) to the statistical uncertainty to give the error-bars depicted in Fig. 2. 
$19^{\text {th }}$ International IUPAP Conference on Few-Body Problems in Physics

\section{Summary and Outlook}

I have reported on an ab-initio calculation of the spectrum of the light pseudoscalar and vector mesons and octet and decuplet baryons in QCD with fully controlled systematics, as performed by the Budapest-Marseille-Wuppertal collaboration [15]. The outcome is in perfect agreement with experiment. As a result we can claim to understand the origin of the bulk of the visible mass in the universe quantitatively and from first principles; it is due to the dynamical mechanism which binds almost-massless quarks and exactly-massless gluons into protons and neutrons, thereby giving them a mass of order $1 \mathrm{GeV}$ each.

In my view this is more of a beginning rather than the end. Within QCD there is still a number of challenges. One of them is to obtain similarly precise results for the mass and the decay width of states which decay under strong interactions, even though the pertinent conceptual questions have been solved $[29,30]$.

By the general philosophy that "yesterdays discovery is todays high-precision measurement and tomorrows background", we are now in an era where large-scale lattice QCD simulations are used to separate QCD effects from the electroweak observables we are interested in. Typical examples include $f_{K} / f_{\pi}, f_{D_{s}} / f_{D}, B_{K}, B_{B}$ and various form factors between pions, kaons, and $B$ and $D$ mesons, since these QCD matrix elements are needed for a precise determination of various Standard Model parameters, including the CKM matrix elements. In particular, tests of the unitarity of this matrix have the potential to establish stringent bounds on the flavor structure of possible extensions of the Standard Model; see e.g. [31] for an overview. Last but not least, there is the possibility that the reality beyond the Standard Model itself may contain some strongly interacting degrees of freedom.

\section{Acknowledgments}

I'm indebted to all members of the Budapest-MarseilleWuppertal collaboration for the work I'm reporting on. In addition, I would like to thank the staff of the Jülich Supercomputing Center, which is the place where the largest portion of the simulations was performed. For a detailed acknowledgment of all relevant research grants, see the original publication [15].

\section{References}

1. H. Fritzsch, M. Gell-Mann and H. Leutwyler, Phys. Lett. B 47, 365 (1973).

2. D. J. Gross and F. Wilczek, Phys. Rev. Lett. 30, 1343 (1973).

3. H. D. Politzer, Phys. Rev. Lett. 30, 1346 (1973).

4. K. G. Wilson, Phys. Rev. D 10, 2445 (1974).

5. K. G. Wilson, New Phenomena In Subnuclear Physics. Part A. Proceedings of the First Half of the 1975 International School of Subnuclear Physics, Erice, Sicily, July 11 - August 1, 1975, ed. A. Zichichi, Plenum Press, New York, 1977.
6. H. B. Nielsen and M. Ninomiya, Nucl. Phys. B $\mathbf{1 8 5}$, 20 (1981) [Erratum-ibid. B 195, 541 (1982)].

7. H. B. Nielsen and M. Ninomiya, Nucl. Phys. B 193, 173 (1981).

8. J. B. Kogut, Rev. Mod. Phys. 55, 775 (1983).

9. M. Creutz, Phys. Rev. D 21, 2308 (1980).

10. K. Symanzik, Nucl. Phys. B 226, 187 (1983).

11. K. Symanzik, Nucl. Phys. B 226, 205 (1983).

12. M. Lüscher and P. Weisz, Phys. Lett. B 158, 250 (1985).

13. B. Sheikholeslami and R. Wohlert, Nucl. Phys. B 259, 572 (1985).

14. C. Morningstar and M. J. Peardon, Phys. Rev. D 69, 054501 (2004) [hep-lat/0311018].

15. S. Dürr et al. [Budapest-Marseille-Wuppertal Collab.], Science 322, 1224 (2008), including "supplementary online matrerial" [0906.3599, hep-lat].

16. S. Duane, A. D. Kennedy, B. J. Pendleton and D. Roweth, Phys. Lett. B 195, 216 (1987).

17. T. A. DeGrand and P. Rossi, Comput. Phys. Commun. 60, 211 (1990).

18. J. C. Sexton and D. H. Weingarten, Nucl. Phys. B 380, 665 (1992).

19. M. Hasenbusch, Phys. Lett. B 519, 177 (2001) [heplat/0107019].

20. T. Takaishi and P. de Forcrand, Phys. Rev. E 73, 036706 (2006) [hep-lat/0505020].

21. M.A. Clark and A.D. Kennedy, Phys. Rev. Lett. 98 (2007) 051601 [hep-lat/0608015].

22. S. Dürr et al. [Budapest-Marseille-Wuppertal Collab.], Phys. Rev. D 79, 014501 (2009) [0802.2706, hep-lat].

23. C. Urbach, K. Jansen, A. Shindler and U. Wenger, Comput. Phys. Commun. 174, 87 (2006) [hep-lat/ 0506011].

24. S. Aoki et al. [PACS-CS Collaboration], 0807.1661 [hep-lat].

25. G. Colangelo, S. Dürr and C. Haefeli, Nucl. Phys. B 721, 136 (2005) [hep-lat/0503014].

26. A. Ali Khan et al. [QCDSF-UKQCD Collaboration], Nucl. Phys. B 689, 175 (2004) [hep-lat/0312030].

27. C. Aubin et al. [MILC Collaboration], Phys. Rev. D 70, 114501 (2004) [hep-lat/0407028].

28. P. Langacker and H. Pagels, Phys. Rev. D 10, 2904 (1974).

29. M. Lüscher, Nucl. Phys. B 364, 237 (1991).

30. V. Bernard, M. Lage, U. G. Meissner and A. Rusetsky, JHEP 0808, 024 (2008) [0806.4495, hep-lat].

31. M. Antonelli et al., arXiv:0907.5386 [hep-ph]. 